\title{
Interface saúde da família \& saúde mental: uma estratégia para o cuidado
}

Interface between the family health program \& mental health assistance: a strategy for care

\author{
Interfaz salud de la familia y salud mental: \\ una estrategia para la atención
}

André Luiz Binotto ${ }^{1 *}$, Luciane Loures dos Santos², Quitéria de Lourdes Lourosa ${ }^{3}$, Sonia Camila Sant'Anna4, Ana Carolina Guidorizzi Zanetti ${ }^{5}$, Aldaísa Cassanho Forster ${ }^{6}$, João Mazzoncini de Azevedo Marques ${ }^{7}$

Palavras-chave: Atenção Primária à Saúde

Saúde Mental Saúde da Família

\section{Resumo}

Estudos internacionais mostram que a Atenção Primária à Saúde (APS) realiza a maioria dos atendimentos psiquiátricos, pois, mesmo nos países que mais investem em saúde mental, existe uma lacuna entre a oferta e a demanda por serviços especializados. No Brasil, preconiza-se que a Estratégia de Saúde da Família (ESF) assista aos pacientes com problemas de saúde mental e 0 apoio matricial é uma ferramenta para qualificar esse trabalho. Objetivamos conhecer o perfil epidemiológico dos usuários atendidos conjuntamente por uma equipe de ESF e sua equipe matriciadora de Saúde Mental, durante dois anos, por meio da revisão dos prontuários desses pacientes. De um total de 203 atendimentos realizados nesse período, 74\% eram de mulheres entre 50 a 59 anos (33\%); os diagnósticos mais comuns foram Episódio Depressivo Maior (61\%), Transtorno de Ansiedade Generalizada (33\%), Esquizofrenia e Transtorno de Estresse Pós-Traumático (11\% cada). Trinta e cinco por cento dos pacientes foram encaminhados, sendo a maioria para psicoterapia (94\%) e o restante para um ambulatório secundário de Saúde Mental (6\%). Os psicofármacos mais prescritos foram os inibidores seletivos da recaptação da serotonina (76\%), anti-histamínicos (37\%), benzodiazepínicos (37\%) e antipsicóticos típicos (26\%). Esta interface representou um acesso facilitado e eficaz para o usuário com transtorno mental de maior gravidade/complexidade e abriu portas para a integralidade da atenção e a interdisciplinaridade no trabalho cotidiano.

\footnotetext{
${ }^{1}$ Departamento de Medicina Social da Faculdade de Medicina de Ribeirão Preto. Universidade de São Paulo (USP). andrebinotto@yahoo.com.br ${ }^{2}$ Departamento de Medicina Social da Faculdade de Medicina de Ribeirão Preto. Universidade de São Paulo (USP). luloures@fmrp.usp.br ${ }^{3}$ Centro de Atenção Primária da Faculdade de Medicina de Ribeirão Preto. Universidade de São Paulo (USP). quitylouro@uol.com.br ${ }^{4}$ Centro de Atenção Primária da Faculdade de Medicina de Ribeirão Preto. Universidade de São Paulo.(USP). scamila29@uol.com.br ${ }^{5}$ Departamento de Enfermagem Psiquiátrica e Ciências Humanas da Escola de Enfermagem de Ribeirão Preto. Universidade de São Paulo (USP). carolzan@hotmail.com

${ }^{6}$ Departamento de Medicina Social da Faculdade de Medicina de Ribeirão Preto. Universidade de São Paulo (USP). acforste@fmrp.usp.br ${ }^{7}$ Programa de Residência de Medicina de Família e Comunidade do Hospital das Clínicas da Faculdade de Medicina de Ribeirão Preto Universidade de São Paulo (USP). jmaq@uol.com.br *Autor correspondente.

Fonte de financiamento: nenhuma. Conflito de interesses: declaram não haver.

Recebido em: 22/02/2012

Aprovado em: 14/04/2012
} 
Keywords:

Primary Health Care

Mental Health

Family Health

Atención Primaria de Salud

Salud Mental

Salud de la Familia

\section{Abstract}

International studies show that the Primary Health Care (PHC) held the majority of psychiatric care, because even in countries that invest more in mental health, there is a gap between supply and demand for specialist services. In Brazil, it is recommended that the Family Health assist patients with mental health problems and the support matrix is an important instrument to qualify this work. We aim to know the epidemiological profile of users attended by Family health service and the mental health team, during two years, through reviewing medical records. Seventy four percent of the 203 consultations were females from 50 to 59 years old (33\%); the main diagnoses were: major depressive episode (61\%), generalized anxiety disorder (33\%), schizophrenia and posttraumatic stress disorder (11\% each). 0 f these, $35 \%$ were referred for psychotherapy (94\%) or to secondary mental health outpatient service (6\%). The most commonly prescribed psychotropic drugs were selective inhibitors of serotonin reuptake (76\%), antihistamines (37\%), benzodiazepines (37\%) and typical antipsychotics (26\%). This interface represented a facilitated and effective access to the user with a mental disorder, and has opened doors to comprehensive health care and interdisciplinary on daily work.

\section{Resumen}

Estudios internacionales muestran que la atención primaria hace la mayoría de los tratamientos psiquiátricos, porque, incluso en los países que más invierten en salud mental, tiene una brecha entre la necesidad y la oferta de los servicios especializados. En Brasil, se preconiza que la Estrategia de Salud de la Familia (ESF) atienda los pacientes con problemas de salud mental y la estrategia de matriciamento constituye una herramienta para calificar el trabajo. Este estudio objetivó analizar el perfil epidemiológico de usuarios atendidos en conjunto por un equipo de ESF y su equipo de Salud Mental, durante el periodo de dos años, a través de la revisión de los prontuarios de los usuarios. De un total de 203 consultas conjuntas en el periodo, 74\% eran de mujeres entre 50 a 59 años (33\%); los diagnósticos más comunes fueran Episodio Depresivo Mayor (61\%), Trastorno de Ansiedad Generalizada (33\%), Esquizofrenia y Trastorno por Estrés Postraumático (11\% cada uno). Treinta y cinco porcentual de los pacientes fueron encaminados, la mayoría fueran para la psicoterapia (94\%) y los demás para uno ambulatorio secundario de salud mental (6\%). Los psicotrópicas más comúnmente recetados fueron los inhibidores selectivos de la recaptación de serotonina (76\%), antihistamínicos (37\%), benzodiacepinas (37\%), y antipsicóticos típicos (26\%). Esta interfaz representó un acceso fácil y eficaz para el usuario con trastorno mental de mayor gravedad/complexidad, abrió las puertas para la integralidad de la atención e interdisciplinaridad en el trabajo cotidiano.

\section{Introdução}

A prevalência de transtornos mentais na população em geral é muito significativa, com a maioria dos estudos epidemiológicos encontrando índices entre $10 \%$ e $20 \%$ em um ano e $20 \%$ e $40 \%$ ao longo da vida ${ }^{1,2}$. No Brasil, segundo dados do Ministério da Saúde, 3\% da população sofre de algum transtorno mental grave ou persistente e aproximadamente $12 \%$ da população necessita, em algum momento, de atendimento contínuo ou eventual em saúde mental ${ }^{3}$. As incapacidades, as consequências econômicas associadas e o comprometimento da qualidade de vida são tão importantes - individual e coletivamente - quanto aquelas associadas às doenças não psiquiátricas, como hipertensão, diabetes, asma, artrite e outras ${ }^{4-6}$. A comorbidade entre transtornos mentais e condições médicas gerais também é comum e leva a comprometimentos mais graves e piores prognósticos de ambas as condiçōes ${ }^{7}$. Além disso, a presença de transtornos mentais inadequadamente manejados dificulta a aderência dos pacientes às condutas preventivas, curativas e reabilitadoras relativas às doenças não psiquiátricas ${ }^{8}$.

Estudos avaliando a prevalência de transtornos mentais em APS - inclusive aqueles realizados no Brasil - têm encontrado que cerca de um quarto a um terço dos pacientes atendidos apresenta ao menos um diagnóstico de transtorno mental conforme a Classificação Estatística Internacional de Doenças e Problemas Relacionados à Saúde CID-10 $10^{1,9}$. Mesmo nos países com sistemas de acesso universal com maior investimento per capita em saúde é na Atenção Primária à Saúde (APS) que é realizada a maioria dos tratamentos de transtornos mentais ${ }^{10-12}$.

No entanto, em média, apenas $50 \%$ desses casos sáo adequadamente diagnosticados e uma percentagem menor ainda é tratada de acordo com as evidências científicas existentes ${ }^{2}$. Adicionalmente, na APS, um número significativo de pessoas recebe tratamento - principalmente através de psicofármacos - quando não apresentam diagnósticos de transtornos mentais justificando tal abordagem?

Nas últimas décadas, a constatação dessa situação estimulou uma série de pesquisas na APS com o intuito de esclarecer quais fatores estáo relacionados ao tratamento de maior ou menor qualidade dos transtornos mentais e quais intervençóes nesse nível de atenção podem levar a um cuidado mais adequado. $\mathrm{O}$ conjunto desses estudos tem sugerido que modelos de cuidado envolvendo colaboração intensiva entre equipes de APS e equipes especializadas de saúde mental - basicamente, uma proximidade maior, cotidiana e contínua - podem estimular abordagens mais adequadas quanto ao tratamento dos transtornos mentais ao longo do tempo ${ }^{9,11-13}$.

No Brasil, a APS está estruturada através da Estratégia de Saúde da Família (ESF), implantada em 1994, que tem como fundamentos a acessibilidade, integralidade da assistência, 
criação do vínculo com a comunidade adscrita, capacitação constante do profissional, avaliação do serviço, associado à participação popular e ao controle social ${ }^{3}$.

Assumindo seu papel de prover atenção integral à saúde, a ESF é também uma ótima ferramenta para trabalhar a saúde mental na comunidade - incluindo o tratamento dos transtornos mentais - pois, através de seus fundamentos, pode identificar as principais dificuldades e fatores estressores no ambiente familiar e comunitário, além de propiciar um seguimento mais próximo do usuário e da comunidade.

O apoio matricial é uma forma de organizar e ampliar a oferta de açóes em saúde, que lança mão de saberes e práticas especializadas, sem que o usuário deixe de ser cliente da equipe de referência. Dessa maneira, a estratégia do apoio especializado matricial em Saúde Mental às equipes de APS vem ao encontro dos princípios da ESF do ponto de vista de prover uma atenção integral à saúde, facilitando o acesso do usuário à especialidade necessária sem perder a continuidade do tratamento nem o vínculo com a equipe da APS ${ }^{14}$.

Existem poucas experiências nacionais descritas, com pelo menos alguns anos de existência, de colaboração intensiva entre equipes da ESF e equipes de saúde mental. Assim, o objetivo do presente estudo é conhecer o perfil epidemiológico dos usuários atendidos em uma unidade de saúde da família em conjunto com a equipe de Saúde Mental, as principais condutas tomadas nesses atendimentos, dentro de um modelo de colaboração intensiva.

\section{Metodologia}

\section{Contexto do estudo}

Em Ribeirão Preto, interior de São Paulo, a ESF iniciou-se no ano de 1999 com a inauguração do primeiro Núcleo de Saúde da Família (NSF) pela Faculdade de Medicina de Ribeirāo Preto da Universidade de São Paulo - FMRP-USP ${ }^{15}$. Atualmente são cinco equipes de saúde da família vinculadas à FMRP-USP nos moldes preconizados pelo Ministério da Saúde, que têm como objetivo a assistência à população de seu território de abrangência, constituído por cerca de 800-1000 famílias por NSF, totalizando cerca de 20.000 pessoas.

Inicialmente os NSF foram criados para atender à necessidade de campo de ensino em atenção básica e saúde da família do Programa de Residência Médica em Medicina de Família e Comunidade do Hospital das Clínicas da FMRP-USP, e dos cursos de graduaçáo da área da saúde, incluindo medicina, enfermagem, odontologia, farmácia, psicologia, fisioterapia, fonoaudiologia, terapia ocupacional, nutrição e metabolismo, e informática biomédica ${ }^{15}$. Atualmente os NSF são unidades de saúde integradas à Secretaria Municipal de Saúde de Ribeirão Preto.

Desde 1999, as equipes da ESF realizam discussôes clínicas na própria unidade com a participação de especialistas das áreas de Clínica Médica, Pediatria, Ginecologia e Saúde Mental; posteriormente, outras áreas foram incluídas neste processo como oftalmologia e dermatologia. Esta prática surgiu com o intuito de aumentar a resolubilidade da atenção, promover a educação permanente da equipe e dos estudantes de graduação e pós-graduação. No entanto, ao longo dos anos, foi apenas na interface com a Saúde Mental que se desenvolveu explicitamente um modelo de colaboração intensiva, com objetivos de assistência, ensino e pesquisa.

Uma equipe de saúde mental - constituída por um psiquiatra supervisor, uma enfermeira especialista em saúde mental e médicos residentes do programa de psiquiatria do Hospital das Clínicas da FMRP-USP - tem encontros semanais com duração de 90 minutos nas próprias unidades de Saúde da Família, com a participação do médico de família, da equipe de enfermagem, dos residentes de Medicina de Família e Comunidade-MFC e agentes comunitários de saúde, além de alunos de graduaçáo e pós-graduação em estágio. As atividades desenvolvidas nesses encontros incluem discussões de temas e de casos em saúde mental, atendimentos em conjunto pela equipe de ESF e equipe matriciadora (incluindo visitas domiciliares), além da discussão/reflexão sobre aspectos organizacionais e de funcionamento cotidiano do serviço. A seleçấo dos casos a serem discutidos e/ou atendidos conjuntamente é feita pela equipe de ESF, conforme as demandas surgidas no seu trabalho cotidiano, priorizando-se aqueles de maior gravidade/complexidade e que necessitam de avaliaçáo especializada mais rapidamente,

Depois de cada atendimento conjunto, é definido consensualmente qual será a continuaçáo do tratamento - se o atendimento conjunto será mantido, se o paciente será acompanhado apenas pelos profissionais da ESF ou se o caso necessita de encaminhamento para atendimento especializado. Esse atendimento especializado pode ser realizado pelos próprios médicos psiquiatras ou com a enfermeira especializada que participam dos encontros, ou com outros profissionais que atuam em outros níveis do sistema de saúde.

\section{Sujeitos}

Este relato descreve o perfil dos usuários atendidos no período de agosto de 2007 a agosto de 2009 pela equipe de Saúde da Família em conjunto com a equipe de Saúde Mental em um NSF.

Não foram incluídos os casos que necessitaram apenas de discussão com a equipe matricial nos encontros semanais e os casos dos usuários que mudaram da área de abrangência. 


\section{Coleta de dados}

Foi realizado um estudo retrospectivo de desenho transversal, cujos dados foram gerados através de levantamento de anotaçôes contidas nos prontuários individuais dos usuários com transtornos mentais atendidos em conjunto com a equipe de apoio matricial em Saúde Mental. O diagnóstico dos transtornos mentais foi dado a princípio pelo médico de família e pelos residentes de MFC da equipe de Saúde da Família e, posteriormente, confirmado pela equipe de apoio matricial. A equipe médica da saúde da família foi treinada pela equipe de apoio matricial para utilizar o Mini International Neuropsychiatric Interview (MINI), uma entrevista semiestruturada para diagnosticar transtornos mentais, que foi traduzido e validado no Brasil para utilização na APS ${ }^{16}$.

Foram coletadas e analisadas as seguintes variáveis: faixa etária distribuída conforme o Sistema de Informaçáo da Atenção Básica (SIAB), sexo, doenças mais prevalentes, medicamentos mais prescritos, número de consultas por pacientes e número de encaminhamentos. Para a realização da coleta, criamos um instrumento com o intuito de registrar tais variáveis.

Estes dados fazem parte do projeto de pesquisa: "Perfil Epidemiológico de pacientes com transtornos mentais atendidos em um Núcleo de Saúde da Família vinculado à Faculdade de Medicina de Ribeiráo Preto", que foi submetido ao Comitê de Ética em Pesquisa do Centro de Saúde Escola Dr. Joel Domingos Machado da FMRP-USP, que aprovou o projeto em 22 de julho de 2010 (Protocolo no 376/ CEP-CSE-FMRP-USP).

\section{Análise dos resultados}

Os dados foram digitados em uma planilha do Excel, simultaneamente com a coleta. Depois de toda a coleta e digitaçáo dos dados, foi criada uma máscara do banco de dados no programa Epi Info 6 para análise e interpretação dos dados. Realizamos uma análise descritiva dos resultados encontrados nos prontuários.

\section{Resultados}

No período estudado, foram realizados 203 atendimentos conjuntos entre a equipe de saúde mental e a equipe da ESF. Participaram destes atendimentos 68 usuários, sendo que 22 foram excluídos da pesquisa, pois mudaram da área e seus prontuários foram transferidos, dificultando o acesso aos dados.

Ocorreram, em média, 3,2 consultas por paciente, sendo que quatro pacientes tiveram entre oito e nove consultas conjuntas; eles apresentavam os diagnósticos de Esquizofrenia, Episódio Depressivo Maior (EDM), Retardo mental e Transtorno de Ansiedade Generalizada (TAG). Outros 13 usuários participaram apenas uma vez do atendimento conjunto, pois foram encaminhados para profissionais especializados ou faltaram ao atendimento agendado. Outro fato a ser considerado é que, em alguns casos, um atendimento conjunto foi suficiente e esses usuários continuaram o acompanhamento apenas com profissionais da ESF.

Encontramos um predomínio de mulheres (74\%) sobre os homens (26\%) entre os usuários atendidos. Quanto à idade, a faixa etária mais frequente foi entre 50 e 59 anos (33\%), seguida de pacientes com 20 a 39 anos $(24 \%)$ e maiores de 60 (22\%), conforme Figura 1.

A principal doença encontrada foi EDM (61\%), seguida de TAG (33\%), Esquizofrenia e Transtorno de Estresse Pós-Traumático (TEPT) com $11 \%$ de frequência cada patologia, conforme Figura 2.

\section{Atendimento em saúde mental segundo faixa etária}
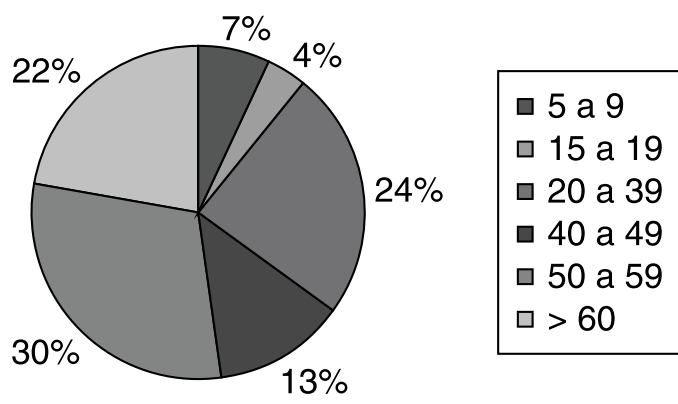

Figura 1. Faixa etária dos usuários atendidos pela equipe matricial em um NSF.

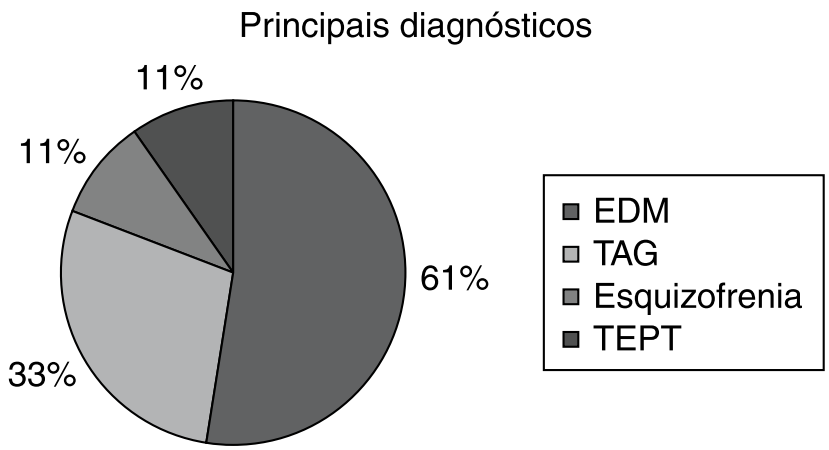

Figura 2. Principais diagnósticos encontrados durante 0 atendimento conjunto. 
Foram encontrados também casos de Transtorno de Personalidade Emocionalmente Instável (TPEI), Transtorno Obsessivo Compulsivo (TOC), TDAH, Retardo Mental, Transtorno de ajustamento, agressividade, fibromialgia e alcoolismo.

Destacamos que 35\% dos casos investigados receberam mais de um diagnóstico, sendo a associação mais frequente o EDM e TAG (15\%), seguida de EDM e TEPT (6\%).

A distribuição das patologias variou segundo o sexo e a faixa etária. Entre os usuários com 5 e 9 anos, a maioria era do gênero masculino com diagnósticos de EDM e sintomas de agressividade, ao contrário dos maiores de 60 anos, em que $87 \%$ eram mulheres com diagnóstico de Episódio Depressivo. Entre os dois usuários atendidos com idade de 15 a 19 anos, um menino apresentava esquizofrenia e a outra usuária foi diagnosticada com o Transtorno de Déficit de Atenção/Hiperatividade (TDAH). A partir dos 20 anos, o gênero feminino passou a ser predominante em todas as idades e o diagnóstico mais encontrado foi o EDM e o TAG.

Também houve diferença entre os diagnósticos segundo o gênero, $80 \%$ dos casos de esquizofrenia foram diagnosticados no sexo masculino e 93\% dos casos de Episódio Depressivo, nas mulheres. Entre os homens, os diagnósticos mais encontrados foram a esquizofrenia (33\%), seguidos do TAG (25\%) e EDM (17\%). Já nas mulheres, $76 \%$ foram diagnosticadas com EDM e $35 \%$ apresentaram TAG, sendo que metade delas apresentava dois ou mais diagnósticos psiquiátricos comórbidos (Figura 3).

As classes terapêuticas mais prescritas foram os inibidores seletivos da recaptação da serotonina-ISRS (76\%), anti-histamínicos (37\%), benzodiazepínicos-BZD (37\%) e antipsicóticos típicos-AP Típicos (26\%). Dos 46 pacientes atendidos $7 \%$ não faziam uso de nenhuma medicação e $11 \%$ usavam apenas ISRS, no entanto, para $74 \%$, foi indicada mais de uma medicação, sendo as associaçōes mais frequentes, o ISRS com anti-histamínicos ou BZD, ou com ambos (Tabela 1).

Três pacientes tiveram indicação de até quatro drogas durante os dois anos de estudo, sendo os ISRS em associação com benzodiazepínico, anti-histamínico e antipsicótico ou antidepressivo tricíclico. Esses usuários tinham os seguintes diagnósticos: TAG; EDM/TAG; e EDM/TEPT; e todos foram encaminhados para o ambulatório secundário de Saúde Mental ou para apoio psicoterápico.

Dos pacientes seguidos, $35 \%$ foram encaminhados: destes, $94 \%$ para psicoterapia e $6 \%$ para um ambulatório secundário de Saúde Mental. O diagnóstico de EDM isolado ou associado à outra patologia foi responsável pelo encaminhamento de $60 \%$ dos casos para psicoterapia.

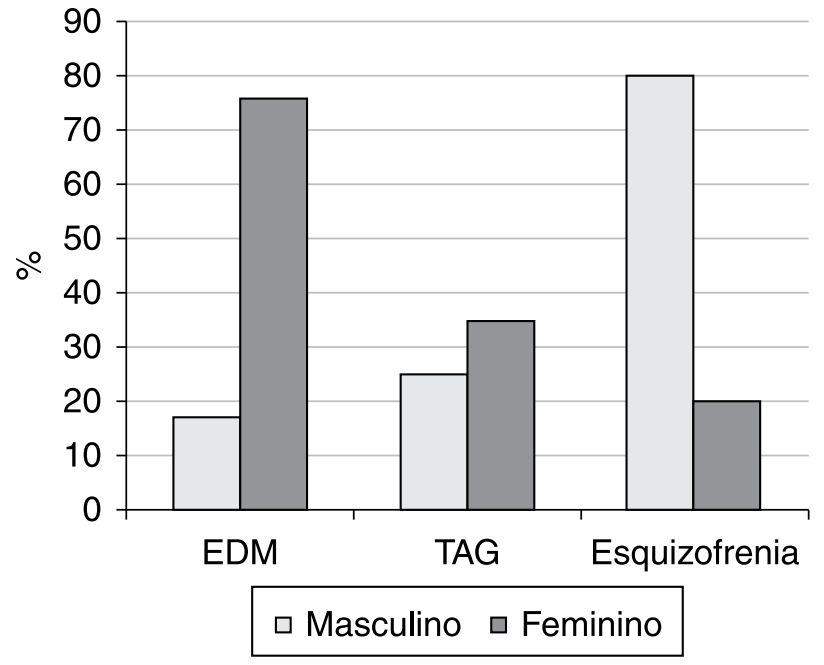

Figura 3. Distribuição dos diagnósticos segundo o gênero.

Tabela 1. Apresentação das medicações prescritas e das principais associações ( $n=46)$. Ribeirão Preto, 2012.

\begin{tabular}{lc}
\hline \multicolumn{1}{c}{ Medicações prescritas } & $\mathrm{n}(\%)$ \\
\hline ISRS & $35(76)$ \\
Antidepressivo tricíclico & $7(15)$ \\
AP Típico & $12(26)$ \\
AP Atípico & $4(9)$ \\
Anti-histamínico & $17(37)$ \\
BZD & $17(37)$ \\
\hline Principais associações prescritas & \\
\hline ISRS + Anti-histamínico & $6(13)$ \\
ISRS + BZD & $5(11)$ \\
ISRS + Anti-histaminíco + BZD & $5(11)$ \\
ISRS + AP Típico & $3(6)$ \\
\hline
\end{tabular}

\section{Discussão e conclusões}

De acordo com os dados encontrados, observamos que a maioria dos usuários participantes das atividades de atendimento conjunto pela equipe de ESF e equipe matriciadora tinha ao menos um diagnóstico em saúde mental e recebia tratamento medicamentoso adequado.

Essa interface possibilitou a avaliação e tratamento de casos de transtornos mentais considerados mais graves-complexos pela equipe de ESF e auxiliou na sua capacitação para manejá-los, provavelmente ajudando a evitar o sentimento de despreparo e fragilidade perante os problemas de saúde mental táo comumente descrito entre as equipes de APS ${ }^{17,18}$.

Essa experiência de colaboração intensiva precede a normatizaçáo das atividades dos NASF (Núcleo de Apoio à Saúde da Família) pelo Ministério da Saúde em 2008 que tem como objetivo: 
[...] ampliar a abrangência e o escopo das ações da atenção básica, bem como sua resolubilidade, apoiando a inserção da estratégia de Saúde da Família na rede de serviços e o processo de territorialização e regionalizaçáo a partir da atenção básica ${ }^{19}$.

E está em conformação com a estratégia de humanização da assistência ao usuário que o MS conceitua em 2004 como Equipe Matricial:

[...] que lança mão de saberes e práticas especializadas, sem que o usuário deixe de ser cliente da equipe de referência (equipe da ESF).

A equipe de referência e o apoio matricial, juntos, permitem um modelo de atendimento voltado para as necessidades de cada usuário: as equipes conhecem os usuários que estấo sob o seu cuidado e isso favorece a construção de vínculos terapêuticos e a responsabilização (definição de responsabilidades) das equipes.

Permite ainda a expressão dos saberes, desejos e práticas dos profissionais, bem como um melhor acompanhamento do processo saúde/doença/intervenção de cada sujeito-usuário ${ }^{20}$.

Esses modelos de colaboraçáo intensiva implicam tanto mudanças internas em cada serviço como no modo desses serviços se relacionarem (sua interface), ou seja, implicam uma mudança na organização do sistema de saúde de referência de uma determinada população. Assim, estudos têm sugerido que os seguintes elementos são importantes para a organização de uma colaboração adequada entre APS e serviços especializados de saúde mental: acompanhamento longitudinal pelos mesmos profissionais de APS e de Saúde Mental; estrutura física e organizacional (incluindo tempo suficiente) para a equipe de APS realizar adequadamente rastreamento, diagnóstico, tratamento e encaminhamento para serviços especializados (quando necessário); acesso às diretrizes de tratamento baseadas em evidência científica adequada para o trabalho em APS (incluindo instrumentos padronizados de apoio para o diagnóstico e monitoramento do resultado do tratamento); acesso à consultoria especializada no serviço de APS para esclarecer dúvidas e tomar decisóes quanto ao tratamento, quando necessário (incluindo a definiçâao dos papéis da APS e da Atençáo Especializada em casos de maior gravidade/complexidade); possibilidade dos profissionais de cuidados primários avaliarem e acompanharem casos específicos junto com profissionais especializados no próprio serviço de APS; disponibilidade e agilidade no acesso aos tratamentos oferecido pelos profissionais/serviços especializados para os casos de maior gravidade/complexidade ${ }^{9,12,13}$.

Organizada desse modo, a colaboração entre os níveis de atençâo facilita tanto a disponibilidade de cuidados para cada indivíduo como o uso mais eficiente dos recursos do sistema de saúde coletivamente, através da organizaçáo do atendimento em etapas específicas (stepped-care na literatura internacional), conforme diretrizes previamente pactuadas e constantemente atualizadas e flexibilizadas conforme a necessidade, nos espaços e tempos de encontros das equipes de APS e Especializadas em Saúde Mental. Potencialmente, pode tanto evitar o acúmulo de casos de pouca complexidade/gravidade nos serviços especializados, como permitir acesso rápido e ágil aos tratamentos que só podem ser realizados por profissionais especializados para casos/situações de maior complexidade/gravidade ${ }^{9,12,13}$.

Assim, a descrição dessa experiência pode ajudar na organização detalhada da interface saúde da família/saúde mental em outros sistemas locais de saúde, possibilitando incluir, desde o início, os componentes estruturais necessários para o seu bom desenvolvimento. É estimulante constatar que, com essa organização do atendimento, mesmo nesse grupo de pacientes com transtornos mentais de grande complexidade/gravidade, apenas cerca de um terço necessitou de acompanhamento em serviço especializado de saúde mental. Tal resultado confirma um estudo anterior que encontrou, nos pacientes acompanhados por outra equipe de ESF ligada ao Centro de Saúde Escola Dr. Joel Machado da FMRP-USP, uma resolutividade de aproximadamente $90 \%$, quando considerados todos os pacientes diagnosticados com transtorno mental em um período de 7 meses ${ }^{19}$.

Uma limitação identificada nas atividades desenvolvidas foi à indisposição de alguns pacientes em participar do atendimento conjunto pela presença de vários profissionais na sala de atendimento (médico, enfermeiro, psicólogo, residentes, alunos de graduação). Além disso, cabe ressaltar que os estudos foram realizados em unidades de saúde vinculadas a uma universidade pública que dispóe dessa atividade de matriciamento em saúde mental e que náo representa a realidade do município.

Dentro da universidade, esse modelo de colaboraçáo intensiva também pode colaborar para a formação adequada em Saúde Mental/Psiquiatria, tanto na graduação médica como na residência de Medicina de Família e Comunidade, conforme descrito em outros trabalhos, através do oferecimento de uma educação permanente adequada às necessidades $\mathrm{e}$ demandas encontradas em APS ${ }^{16,21,22}$. Mas não deveriam limitar-se exclusivamente a unidades de saúde atreladas ao ensino, uma vez que a interface entre a Saúde da Família e a Saúde Mental representa um importante instrumento para a integralidade do cuidado.

Mesmo apesar de algumas limitaçôes, como a indisposição que alguns pacientes encontram em um atendimento com vários profissionais (médico, enfermeiro, psicólogo, residentes, 
alunos de graduação) e muitas vezes confundir a demanda daquela consulta (saúde mental ou outra condição médica geral), além da dificuldade de organizar e reservar um espaço na agenda da unidade para contemplar os atendimentos conjuntos em meio às várias demandas do cotidiano da atenção primária, os resultados ilustram que a interface entre a Saúde da Família e a Saúde Mental representa um importante instrumento para a integralidade do cuidado.

\section{Referências}

1. Kessler RC, Aguilar-Gaxiola S, Alonso J, Chatterji S, Lee S, Ormel $J$, et al. The global burden of mental disorders: an update from the WHO World Mental Health (WMH) surveys. Epidemiol Psichiatr Soc. 2009; 18(1): 23-33. PMid:19378696 PMCid:3039289.

2. Vazquez-Barquero JL, Herran A, Simon JA. Epidemiology of mental disorders in the community and primary care. In: Tansella M, Thornicroft $G$, editors. Common Mental Disorders in Primary Care. London: Routledge; 1999. p. 3-16.

3. Brasil. Ministério da Saúde. Política Nacional de Saúde Mental [online]. Brasília. [acesso em 2008 Set]. Disponível em: http://portaldasaúde. gov.br

4. Eaton WW, Martins SS, Nestadt G, Bienvenu OJ, Clarke D, Alexandre P. The burden of mental disorders. Epidemiol Rev. 2008; 30: 1-14. PMid:18806255 PMCid:2683377. http://dx.doi.org/10.1093/epirev/ $\mathrm{mxn011}$

5. Jenkins $R$, Singh $B$. Mental disorder and disability in the population. In: Thornicroft G, Szmukler G, editors. Textbook of Community Psychiatry. Avon: Oxford University Press; 2001. p. 101-16.

6. Knapp M. The costs of mental disorders. In: Thornicroft G, Szmukler G, editors. Textbook of Community Psychiatry. Avon: Oxford University Press; 2001. p. 129-40.

7. Scott KM, Von Korff M, Alonso J, Angermeyer MC, Bromet E, Fayyad J, et al. Mental-physical co-morbidity and its relationship with disability: results from the World Mental Health Surveys. Psychol Med. 2009; 39(1): 33-43. PMid:18366819 PMCid:2637813. http:// dx.doi.org/10.1017/S0033291708003188

8. World Health Organizatio - WHO. Adherence to long-term therapies: evidence for action. Geneva: WHO; 2003.

9. World Health Organization - WHO, World Organization of Family Doctors - WONCA. Integrating mental health in primary care: a global perspective. WHO; WONCA; 2008.

10. Wang PS, Aguilar-Gaxiola S, Alonso J, Angermeyer MC, Borges G, Bromet EJ, et al. Use of mental health services for anxiety, mood, and substance disorders in 17 countries in the WHO world mental health surveys. Lancet. 2007; 370(9590): 841-50. http://dx.doi.org/10.1016/ S0140-6736(07)61414-7

11. World Health Organizatio - WHO. Mental health: new understanding, new hope. Geneva: WHO; 2001. The World Health Report 2001. PMid:12053639 PMCid:2214050.

12. Kates N, Craven M, Collaborative Working Group of the College of Family Physicians of Canada, Canadian Psychiatric Association. Shared mental health care. Update from the Collaborative Working Group of the College of Family Physicians of Canada and the Canadian Psychiatric Association. Can Fam Physician. 2002; 48: 936. PMid:16399961.

13. Unutzer J, Schoembaum M, Druss BG, Katon WJ. Transforming mental health care at interface with general medicine: report for the President's commission. Psychiatr Serv. 2006; 57(1): 37-47. http:// dx.doi.org/10.1176/appi.ps.57.1.37

14. Brasil. Ministério da Saúde. Secretaria de Atenção à Saúde. Coordenação de SM e Coordenação da Gestão da AB. SM e AB. O vinculo e o diálogo necessários - Inclusão das ações de SM na AB. Brasília; 2003. Circular conjunta n. 01/03 de 13/11/2003.

15. Caccia-Bava MC, Forster AC, Laprega MR, Silva AS, Santos LL. A Atenção Básica à Saúde e a estratégia Saúde Familiar. In: Santoro JR. Ecologia e Desenvolvimento Humano. Ribeirão Preto: FUNPEC Editora; 2008. p. 253-68. PMid:18585532.

16. De Azevedo Marques JM, Zuardi AW. Validity and applicability of the Mini International Neuropsychiatric Interview administered by family medicine residents in primary health care in Brazil. Gen Hosp Psych. 2008; 30: 303-310. http://dx.doi.org/10.1016/j. genhosppsych.2008.02.001

17. Figueiredo MD, Campos RO. Saúde Mental e Atenção Básica à Saúde: o apoio matricial na construção de uma rede multicêntrica. Saúde em Debate. 2008; 32(78-80): 143-149.

18. Schneider ARS. A rede de atenção em saúde mental: a importância da interação entre a atenção primária e os serviços de saúde mental. Rev Cienc Saude. 2009; 2(2): 78-84.

19. Brasil. Portaria no 154, de 24 de janeiro de 2008. Cria os Núcleos de Apoio à Saúde da Família - NASF. Diário Oficial da União, Brasília, 24 jan. 2008. Seção 1, n. 18, p. 47-49.

20. Brasil. Ministério da Saúde. Secretaria-Executiva. Núcleo Técnico da Política Nacional de Humanização. HumanizaSUS: equipe de referência e apoio matricial. Brasília: Ministério da Saúde; 2004.

21. Figueiredo MAC, Forster AC. Percepção e avaliação dos alunos do curso de medicina de uma escola médica pública sobre a importância do estágio em saúde da família na sua formação. Rev Bras Educ Med. 2008; 32(1): 83-89. PMid:11578983.

22. Hodges B, Inch C, Silver I. Improving the psychiatric knowledge, skills, and attitudes of primary care physicians, 1950-2000: a review. Am J Psychiatr. 2001; 158(10): 1579-86. http://dx.doi.org/10.1176/appi. ajp.158.10.1579 